\title{
ERRATUM
}

\section{Erratum to: Racial Segregation, Income Inequality, and Mortality in US Metropolitan Areas}

\author{
Amani M. Nuru-Jeter and Thomas A. LaVeist
}

The first two paragraphs of the article as published require some correction and should read as follows:

Evidence of the relationship between income inequality and mortality in the United States has been mixed. The majority of the published research shows a positive income inequality/mortality association. ${ }^{1-15}$ However, there is also evidence suggesting that the association between income inequality and mortality is conditional on other factors such as racial density. ${ }^{16-20}$ Further, income inequality in the United States largely reflects income inequality between racial groups as the bifurcation of the middle class along with patterns of racial residential segregation has resulted in racial differences in the concentration of poverty and affluence between racial groups with blacks experiencing higher levels of concentrated poverty compared to whites. ${ }^{17,21-23}$ This has to do both with existing patterns of racial segregation and the higher proportion of low-income blacks relative to low-income whites.

In spite of this phenomenon, little has been done to examine how the income inequality/mortality relationship varies as a function of race, racial density, or racial segregation. Some studies have adjusted for \% black, but results have been mixed. Whereas some studies show a significant positive association between income inequality and mortality after adjusting for \% black, others show that after adjusting for \% black, income inequality is no longer significantly associated with mortality. Still others show a protective effect of income inequality after adjusting for \% black. ${ }^{17,24-29}$ Income inequality and mortality are both higher among blacks than among whites. ${ }^{11,19,30}$ Income inequality is also related to growing spatial separation between blacks and whites. ${ }^{17,22,23}$ However, it is unclear whether and to what extent racial segregation impacts the association between income inequality and mortality. Understanding these associations may help elucidate the mechanisms driving the association between income inequality and mortality both between and within racial groups in the United States.

Nuru-Jeter is with the Divisions of Community Health and Human Development, and Epidemiology, School of Public Health, University of California Berkeley, Berkeley, CA, USA; LaVeist is with the Department of Health Policy and Management, Bloomberg School of Public Health, Johns Hopkins University, Baltimore, MD, USA.

Correspondence: Amani M. Nuru-Jeter, Divisions of Community Health and Human Development, and Epidemiology, School of Public Health, University of California Berkeley, Berkeley, CA, USA. (E-mail: anjeter@berkeley.edu)

The online version of the original article can be found at http://dx.doi.org/10.1007/s11524-010-9524-7. 